Acta Crystallographica Section C

Crystal Structure

Communications

ISSN 0108-2701

\title{
2-[1-(Thiosemicarbazono)ethyl]pyridinium Chloride
}

\section{S. Abram and U. Abram}

This electronic document was scanned from an archival copy of material deposited to accompany a paper published in an IUCr journal. In many cases the only accessible copy was a microfilm of a poor-quality original. 


$$
83435
$$


$\begin{array}{rrrrrr}4 & 8 & 3 & 163 & 167 & 6 \\ -2 & 9 & 3 & 39 & 30 & 11\end{array}$

$\begin{array}{rrrrrr}-1 & 9 & 3 & 39 & 30 & 11 \\ -1 & 9 & 3 & 73 & 66 & 4 \\ 0 & 9 & 3 & 0 & 29 & 1\end{array}$

$\begin{array}{rrrrr}1 & 9 & 3 & 224 & 234 \\ -8 & 0 & 4 & 103 & 105\end{array}$

$\begin{array}{lllll}-8 & 0 & 4 & 103 & 105 \\ -6 & 0 & 4 & 199 & 213\end{array}$

$\begin{array}{lllll}-4 & 0 & 4 & 205 & 205\end{array}$

$\begin{array}{rrrrr}-2 & 0 & 4 & 309 & 307 \\ 0 & 0 & 4 & 512 & 551\end{array}$

$\begin{array}{llllll}2 & 0 & \frac{1}{2} & 562 & 597 & 2 \\ 4 & 0 & 4 & 333 & 324 & 3\end{array}$

$\begin{array}{rrrrrr}5 & 0 & 4 & 255 & 258 & 3 \\ 8 & 0 & 4 & 36 & 25 & 17\end{array}$

$\begin{array}{rrrrrr}-9 & 1 & 1 & 34 & 39 & 22 \\ -5 & 1 & 4 & 98 & 96 & 5\end{array}$

$\begin{array}{rrrrrr}-8 & 1 & 4 & 98 & 96 & 5 \\ -7 & 1 & 4 & 53 & 38 & 8\end{array}$

$\begin{array}{lllll}-6 & 1 & 4 & 137 & 133 \\ -5 & 1 & 4 & 114 & 117\end{array}$

$\begin{array}{llllll}-4 & 1 & 4 & 49 & 46\end{array}$

$\begin{array}{rrrrr}-3 & 1 & 4 & 100 & 98 \\ -2 & 1 & 4 & 209 & 211\end{array}$

$\begin{array}{rrrrrr}-2 & 1 & 4 & 209 & 211 & 3 \\ -1 & 1 & 4 & 510 & 490 & 2 \\ 0 & 1 & 4 & 611 & 511 & 12\end{array}$

$\begin{array}{rrrrrr}1 & 1 & 4 & 611 & 511 & 12 \\ 2 & 1 & 4 & 78 & 78 & 2\end{array}$

$\begin{array}{rrrrr}2 & 1 & 4 & 137 & 139 \\ 3 & 1 & 4 & 87 & 86\end{array}$

$\begin{array}{rrrrr}3 & 1 & 4 & 87 & 86 \\ 4 & 1 & 4 & 39 & 52 \\ 5 & 1 & 4 & 0 & 37\end{array}$

$\begin{array}{llllll}6 & 1 & 4 & 105 & 105 & 4\end{array}$

$\begin{array}{rrrrrr}7 & 1 & 4 & 20 & 9 & 19 \\ 8 & 1 & 4 & 24 & 35 & 23\end{array}$

$\begin{array}{rrrrrr}-8 & 2 & 4 & 63 & 53 & 7 \\ -7 & 2 & 4 & 45 & 53 & 11\end{array}$

$\begin{array}{rrrrrr}-6 & 2 & 4 & 45 & 53 & 11 \\ -6 & 2 & 4 & 51 & 60 & 8\end{array}$

$\begin{array}{rrrrr}-5 & 2 & 4 & 41 & 32 \\ -4 & 2 & 4 & 199 & 192\end{array}$

$\begin{array}{lllll}-3 & 2 & 4 & 368 & 375 \\ -2 & 2 & 4 & 321 & 302\end{array}$

$-124158155$

$\begin{array}{rrrrrr}0 & 2 & 4 & 16 & 38 & 16 \\ 1 & 2 & 4 & 75 & 81 & 3\end{array}$

$\begin{array}{rrrrr}1 & 2 & 4 & 75 & 81 \\ 2 & 2 & 4 & 125 & 124\end{array}$

$\begin{array}{lllll}3 & 2 & 4 & 214 & 212 \\ 4 & 2 & 4 & 110 & 120\end{array}$

$\begin{array}{rrrrrr}5 & 2 & 4 & 42 & \frac{4}{1} 1 & 10 \\ 6 & 2 & 4 & 212 & 225 & 3\end{array}$

$\begin{array}{rrrrrr}6 & 2 & 4 & 212 & 225 & 3 \\ 7 & 2 & 4 & 24 & 26 & 24 \\ 8 & 2 & 4 & 72 & 65 & 7\end{array}$

$\begin{array}{rrrrrr}8 & 2 & 4 & 72 & 65 & 7 \\ -8 & 3 & 4 & 39 & 31 & 15\end{array}$

$\begin{array}{llllll}-7 & 3 & 4 & 39 & 37 & 13\end{array}$

$\begin{array}{rrrrr}-6 & 3 & 4 & 53 & 53 \\ -5 & 3 & 4 & 273 & 275\end{array}$

$\begin{array}{rrrrr}-4 & 3 & 4 & 0 & 6 \\ -3 & 3 & 1 & 43 & 48\end{array}$

$\begin{array}{rrrrr}-2 & 3 & 4 & 241 & 48 \\ -1 & 3 & 4 & 99 & 119\end{array}$

$\begin{array}{lllll}1 & 3 & 4 & 263 & 261\end{array}$

$\begin{array}{lllll}1 & 3 & 4 & 437 & 438 \\ 2 & 3 & 4 & 150 & 146\end{array}$

$\begin{array}{rrrrr}3 & 3 & 4 & 62 & 9 \\ 4 & 3 & 4 & 229 & 226\end{array}$

$\begin{array}{lllll}5 & 3 & 4 & 109 & 109\end{array}$

$\begin{array}{rrrrrr}6 & 3 & 4 & 124 & 120 & \\ 7 & 3 & 4 & 20 & 40 & 1\end{array}$

$\begin{array}{rrrrrr}-8 & 4 & 4 & 0 & 5 & 1 \\ -7 & 4 & 4 & 90 & 85 & 5\end{array}$

$\begin{array}{rrrrrr}-5 & 4 & 4 & 103 & 91 & 4 \\ -5 & 4 & 4 & 18 & 23 & 17\end{array}$

$\begin{array}{rrrrrr}-5 & 4 & 4 & 18 & 23 & 1 \\ -4 & 4 & 4 & 59 & 62 & \\ -3 & 4 & 4 & 220 & 212 & \end{array}$

$\begin{array}{lllll}-3 & 4 & 4 & 220 & 212 \\ -2 & 4 & 4 & 392 & 362\end{array}$

$\begin{array}{lllll}-1 & 4 & 4 & 261 & 258\end{array}$

$044 \quad 380 \quad 359$

$\begin{array}{rrrrr}1 & 4 & \frac{4}{2} & 34 & 11 \\ 2 & 4 & \frac{4}{4} & 420 & 426\end{array}$

$3 \div 4 \quad 156 \quad 154$

$\begin{array}{rrrrrr}4 & 4 & 4 & 40 & 30 & 10 \\ 5 & 4 & 4 & 0 & 20 & 1\end{array}$

$6 \begin{array}{lllll}6 & 4 & 4 & 92 & 88\end{array}$

$\begin{array}{rrrrrr}7 & 4 & 4 & 51 & 48 & 11 \\ -7 & 5 & 4 & 82 & 76 & 6\end{array}$

$\begin{array}{rrrrrr}-5 & 5 & 4 & 94 & 86 & 5 \\ -5 & 5 & 4 & 25 & 10 & 25\end{array}$

$\begin{array}{rrrrrr}-5 & 5 & 4 & 25 & 10 & 25 \\ -4 & 5 & 4 & 21 & 8 & 21 \\ -3 & 5 & 4 & 81 & 76 & 4\end{array}$

$\begin{array}{rrrrrr}-3 & 5 & 4 & 81 & 76 & 4 \\ -2 & 5 & 4 & 28 & 30 & 14\end{array}$

$\begin{array}{rrrrrr}-1 & 5 & 4 & 42 & 35 & 7 \\ 0 & 5 & 4 & 367 & 368 & 2\end{array}$

$\begin{array}{rrrrr}0 & 5 & 4 & 367 & 368 \\ 1 & 5 & 4 & 95 & 99\end{array}$

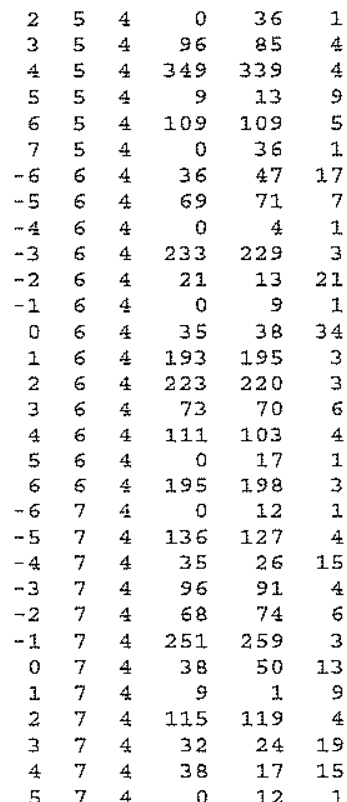

$\begin{array}{rrrrrr}5 & 7 & 4 & 0 & 12 & 1 \\ -4 & 8 & 4 & 0 & 15 & 1\end{array}$

$\begin{array}{rrrrr}-3 & 8 & 4 & 173 & 173 \\ -2 & 8 & 4 & 0 & 13\end{array}$

$\begin{array}{rrrrrr}-2 & 8 & 4 & 0 & 13 & 1 \\ -1 & 8 & 4 & 57 & 50 & 8\end{array}$

$\begin{array}{lllll}0 & 8 & 4 & 117 & 125\end{array}$

$\begin{array}{rrrrrr}1 & 8 & 4 & 0 & 17 & 1 \\ 2 & 8 & 4 & 9 & 9 & 9 \\ 3 & 8 & 4 & 86 & 80 & 6\end{array}$

$\begin{array}{rrrrrr}-2 & 9 & 4 & 21 & 35 & 21\end{array}$

$\begin{array}{rrrrrr}-1 & 9 & 4 & 25 & 20 & 25 \\ 0 & 9 & 4 & 83 & 81 & 3\end{array}$

$\begin{array}{rrrrrr}1 & 9 & 4 & 82 & 81 & 4 \\ -9 & 0 & 5 & 31 & 33 & 23\end{array}$

$\begin{array}{llllll}-7 & 0 & 5 & 202 & 206 & 3\end{array}$

$\begin{array}{lllll}-5 & 0 & 5 & 327 & 334\end{array}$

$\begin{array}{llllll}-3 & 0 & 5 & 395 & 416 & 3 \\ -1 & 0 & 5 & 112 & 115 & 2\end{array}$

$\begin{array}{llllll}1 & 0 & 5 & 595 & 509 & 2 \\ 3 & 0 & 5 & 240 & 235 & 3\end{array}$

$\begin{array}{llllll}5 & 0 & 5 & 219 & 224 & 3\end{array}$

$\begin{array}{rrrrrr}7 & 0 & 5 & 78 & 83 & 6 \\ -9 & 1 & 5 & 51 & 64 & 11 \\ -8 & 1 & 5 & 0 & 8 & 1\end{array}$

$\begin{array}{rrrrrr}-8 & 1 & 5 & 0 & 8 & 1 \\ -7 & 1 & 5 & 161 & 167 & 3\end{array}$

$\begin{array}{rrrrrr}-6 & 1 & 5 & 0 & 40 & 1\end{array}$

$\begin{array}{rrrrrrrrrrrr}-5 & 1 & 5 & 149 & 162 & 2 & -5 & 7 & 5 & 0 & 15 & 1\end{array}$

$\begin{array}{rrrrrrrrrrrr}-4 & 1 & 5 & 164 & 149 & 2 & -4 & 7 & 5 & 0 & 2 & 1 \\ -3 & 1 & 5 & 218 & 206 & 3 & -3 & 7 & 5 & 89 & 88 & 5\end{array}$

$\begin{array}{rrrrrrrrrrrr}-2 & 1 & 5 & 367 & 370 & 2 & -2 & 7 & 5 & 134 & 126 & 3 \\ -1 & 1 & 5 & 514 & 516 & 2 & -1 & 7 & 5 & 39 & 22 & 11\end{array}$

$\begin{array}{rrrrrr}-1 & 1 & 5 & 514 & 516 & 2 \\ 0 & 1 & 5 & 433 & 436 & 10\end{array}$

$\begin{array}{rrrrrr}1 & 1 & 5 & 52 & 51 & 4 \\ 2 & 1 & 5 & 21 & 17 & 20\end{array}$

$\begin{array}{rrrrrr}3 & 1 & 5 & 139 & 130 & 2 \\ 4 & 1 & 5 & 152 & 156 & 2\end{array}$

$\begin{array}{llllll}5 & 1 & 5 & 54 & 54 & 7\end{array}$

$\begin{array}{rrrrrrrrrrrr}6 & 1 & 5 & 130 & 131 & 3 & -4 & 8 & 5 & 0 & 34 & 28\end{array}$

$\begin{array}{rrrrrrrrrrrr}7 & 1 & 5 & 129 & 122 & 4 & -3 & 8 & 5 & 69 & 69 & 7 \\ 8 & 1 & 5 & 60 & 57 & 9 & -2 & 8 & 5 & 25 & 3 & 24 \\ -8 & 2 & 5 & 0 & 36 & 1 & -1 & 8 & 5 & 120 & 126 & \end{array}$

$\begin{array}{rrrrrrrrrrrr}-8 & 2 & 5 & 0 & 36 & 1 & -1 & 8 & 5 & 120 & 126 & 4 \\ -7 & 2 & 5 & 67 & 62 & 6 & 0 & 8 & 5 & 125 & 120 & 3\end{array}$

$\begin{array}{llllll}-6 & 2 & 5 & 46 & 46 & 10\end{array}$

$\begin{array}{rrrrr}-5 & 2 & 5 & 0 & 13 \\ -4 & 2 & 5 & 256 & 250\end{array}$

$\begin{array}{lllll}-3 & 2 & 5 & 196 & 191\end{array}$

$\begin{array}{lllllll}-2 & 2 & 5 & 670 & 644 & 2\end{array}$

$\begin{array}{rrrrrr}2 & 8 & 5 & 86 & 91 & 6 \\ 3 & 8 & 5 & 89 & 78 & 6 \\ -1 & 9 & 5 & 78 & 78 & 4 \\ 0 & 9 & 5 & 44 & 39 & 6 \\ -8 & 0 & 6 & 39 & 24 & 13 \\ -6 & 0 & 6 & 23 & 15 & 23 \\ -4 & 0 & 6 & 730 & 735 & 3 \\ -2 & 0 & 6 & 172 & 171 & 2 \\ 0 & 0 & 6 & 1056 & 1090 & 6\end{array}$

$\begin{array}{rrrrrrrrrrrr}1 & 2 & 5 & 55 & 42 & 3 & -4 & 0 & 6 & 23 & 15 & 23 \\ & 2 & 5 & 570 & 579 & 3 & -4 & 0 & 6 & 730 & 735 & 3\end{array}$

$\begin{array}{llllll}2 & 2 & 5 & 570 & 579 & 3 \\ 3 & 2 & 5 & 156 & 156 & 2\end{array}$

$\begin{array}{lllllll}4 & 2 & 5 & 98 & 100 & 3\end{array}$

$\begin{array}{rrrrrr}5 & 2 & 5 & 118 & 120 & 4 \\ 6 & 2 & 5 & 0 & 33 & 1\end{array}$

$\begin{array}{rrrrrr}7 & 2 & 5 & 0 & 9 & 1 \\ 8 & 2 & 5 & 83 & 91 & 6\end{array}$

$\begin{array}{llllll}-8 & 3 & 5 & 0 & 12 & 1\end{array}$

$\begin{array}{llllll}-7 & 3 & 5 & 13 & 49 & 12 \\ -6 & 3 & 5 & 41 & 37 & 11\end{array}$

$\begin{array}{llllll}1 & 8 & 5 & 0 & 3 & 1 \\ 2 & 8 & 5 & 86 & 91 & 6\end{array}$

$000610561090 \quad 6$

$\begin{array}{rrrrrr}2 & 0 & 6 & 4.9 & 15 & 4 \\ 4 & 0 & 6 & 135 & 136 & 3 \\ 6 & 0 & 5 & 0 & 12 & 1\end{array}$

$\begin{array}{rrrrrr}6 & 0 & 6 & 0 & 12 & 1 \\ 8 & 0 & 6 & 51 & 44 & 11\end{array}$

$\begin{array}{rrrrrr}8 & 0 & 6 & 51 & 44 & 11 \\ -9 & 1 & 6 & 0 & 26 & 1\end{array}$ $\begin{array}{llllllllllll}-5 & 1 & 5 & 275 & 273 & 3 & -3 & 7 & 6 & 170 & 180 & 3\end{array}$

$\begin{array}{rrrrrrrrrrrr}-4 & 1 & 6 & 57 & 57 & 5 & -2 & 7 & 6 & 74 & 89 & 6 \\ -3 & 1 & 6 & 319 & 304 & 3 & -1 & 7 & 6 & 83 & 83 & 5\end{array}$

$\begin{array}{rrrrrrrrrrrr}-2 & 1 & 6 & 967 & 963 & 2 & 0 & 7 & 6 & 4 & 2 & 3\end{array}$

$\begin{array}{llllllllllll}0 & 1 & 5 & 91 & 96 & 2 & 2 & 7 & 6 & 0 & 12 & 1\end{array}$

$\begin{array}{rrrrrrrrrrrr}1 & 1 & 6 & 0 & 3 & 1 & 3 & 7 & 6 & 119 & 118 & 4 \\ 2 & 1 & 6 & 431 & 460 & 3 & 4 & 7 & 6 & 52 & 4.7 & 10\end{array}$

$\begin{array}{rrrrrrrrrrrr}3 & 1 & 6 & 11 & 3 & 10 & -4 & 8 & 6 & 73 & 69 & 7 \\ 4 & 1 & 5 & 63 & 60 & 5 & -3 & 8 & 6 & 43 & 44 & 13\end{array}$

$\begin{array}{llllllllllll}4 & 1 & 6 & 63 & 60 & 5 & -3 & 8 & 6 & 43 & 44 & 13 \\ 5 & 1 & 6 & 87 & 82 & 5 & -2 & 8 & 6 & 46 & 50 & 11\end{array}$

$\begin{array}{rrrrrrrrrrrr}6 & 1 & 6 & 82 & 87 & 5 & -1 & 8 & 6 & 0 & 2 & 1 \\ 7 & 1 & 6 & 74 & 81 & 7 & 0 & 8 & 6 & 122 & 130 & 3\end{array}$

$\begin{array}{rrrrrrrrrrrr}8 & 1 & 6 & 11 & 34 & 10 & 1 & 8 & 6 & 85 & 84 & 5 \\ -8 & 2 & 6 & 40 & 10 & 12 & 2 & 8 & 6 & 49 & 49 & 11\end{array}$

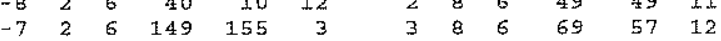

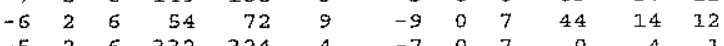

$\begin{array}{rrrrrrrrrrrr}-5 & 2 & 6 & 332 & 324 & 4 & -7 & 0 & 7 & 0 & 4 & 1 \\ -4 & 2 & 6 & 237 & 235 & 3 & -5 & 0 & 7 & 68 & 69 & 5\end{array}$

$\begin{array}{llllllllllll}-3 & 2 & 6 & 149 & 146 & 2 & -3 & 0 & 7 & 521 & 501 & 3\end{array}$

$\begin{array}{llllllllllll}-2 & 2 & 6 & 53 & 52 & 4 & -1 & 0 & 7 & 88 & 93 & 2\end{array}$

$\begin{array}{rrrrrrrrrrrr}-1 & 2 & 6 & 191 & 182 & 2 & 1 & 0 & 7 & 310 & 322 & 3 \\ 0 & 2 & 6 & 260 & 261 & 4 & 3 & 0 & 7 & 376 & 382 & 3\end{array}$

$\begin{array}{llllll}0 & 2 & 6 & 260 & 261 & 4 \\ 1 & 2 & 6 & 122 & 125 & 2\end{array}$

$\begin{array}{llllll}2 & 2 & 6 & 94 & 89 & 3\end{array}$

$\begin{array}{llllll}3 & 2 & 6 & 160 & 151 & 2\end{array}$

$\begin{array}{llllll}4 & 2 & 6 & 211 & 215 & 2 \\ 5 & 2 & 6 & 267 & 268 & 3\end{array}$

$\begin{array}{rrrrrr}6 & 2 & 5 & 48 & 51 & 10\end{array}$

$\begin{array}{rrrrrr}-8 & 3 & 6 & 74 & 71 & 7 \\ -7 & 3 & 6 & 241 & 242 & 3\end{array}$

$\begin{array}{rrrrrr}-7 & 3 & 6 & 241 & 242 & 3 \\ -6 & 3 & 6 & 18 & 19 & 17\end{array}$

$\begin{array}{rrrrrr}-5 & 3 & 6 & 56 & 71 & 6 \\ -4 & 3 & 6 & 148 & 154 & 2\end{array}$

$\begin{array}{rrrrrr}-4 & 3 & 6 & 148 & 154 & 2 \\ -3 & 3 & 5 & 70 & 75 & 4 \\ -2 & 3 & 5 & 184 & 186 & 2\end{array}$

$\begin{array}{rrrrrr}-2 & 3 & 6 & 184 & 186 & 2 \\ -1 & 3 & 6 & 97 & 94 & 2 \\ 0 & 3 & 6 & 204 & 203 & 8\end{array}$

$\begin{array}{llllll}0 & 3 & 6 & 204 & 203 & 8 \\ 1 & 3 & 6 & 184 & 178 & 2\end{array}$

$\begin{array}{rrrrrr}2 & 3 & 6 & 0 & 34 & 1 \\ 3 & 3 & 6 & 327 & 331 & 4\end{array}$

$\begin{array}{rrrrrr}4 & 3 & 6 & 0 & 23 & 1 \\ 5 & 3 & 6 & 81 & 76 & 5\end{array}$

$\begin{array}{rrrrrr}6 & 3 & 6 & 0 & 21 & 1 \\ 7 & 3 & 6 & 170 & 182 & 3\end{array}$

$\begin{array}{rrrrrr}-8 & 4 & 5 & 49 & 34 & 10 \\ -7 & 4 & 6 & 73 & 74 & 7\end{array}$

$\begin{array}{llllll}-7 & 4 & 6 & 73 & 74 & 7 \\ -5 & 4 & 6 & 90 & 92 & 5\end{array}$

$\begin{array}{rrrrrr}-5 & 4 & 6 & 0 & 32 & 1 \\ -4 & 4 & 6 & 221 & 229 & 3\end{array}$

$\begin{array}{rrrrrr}-3 & 4 & 6 & 85 & 90 & 4 \\ -2 & 4 & 6 & 164 & 157 & 2\end{array}$

$\begin{array}{lllll}-2 & 4 & 6 & 164 & 157 \\ -1 & 4 & 6 & 136 & 128\end{array}$

\begin{tabular}{lllll}
0 & 4 & 6 & 230 & 248 \\
\hline & 4 & 4
\end{tabular}

$\begin{array}{rrrrr}1 & 4 & 6 & 0 & 33 \\ 2 & 4 & 6 & 300 & 296\end{array}$

$\begin{array}{rrrrrr}2 & 4 & 6 & 300 & 296 \\ 3 & 4 & 6 & 49 & 46 & 8\end{array}$

$\begin{array}{rrrrrr}4 & 4 & 6 & 103 & 98 & 4 \\ 5 & 4 & 6 & 100 & 100 & 5\end{array}$

$\begin{array}{rrrrrr}6 & 4 & 6 & 52 & 59 & 10 \\ 7 & 4 & 6 & 75 & 66 & 7\end{array}$

$\begin{array}{cccccc}-7 & 5 & 6 & 83 & 86 & 6\end{array}$

$\begin{array}{llllll}-6 & 5 & 6 & 46 & 51 & 11 \\ -5 & 5 & 6 & 25 & 50 & 25\end{array}$

$\begin{array}{rrrrrr}-4 & 5 & 6 & 58 & 60 & 7\end{array}$

$\begin{array}{lllllll}-3 & 5 & 6 & 111 & 112 & 3\end{array}$

$\begin{array}{rrrrrr}-2 & 5 & 6 & 365 & 363 & 4 \\ -1 & 5 & 6 & 0 & 4 & 1\end{array}$

$\begin{array}{llllll}0 & 5 & 6 & 143 & 144 & 2 \\ 1 & 5 & 6 & 146 & 138 & 2\end{array}$

$\begin{array}{llllll}1 & 5 & 6 & 146 & 138 & 2 \\ 2 & 5 & 6 & 149 & 152 & 3\end{array}$

$\begin{array}{llllll}3 & 5 & 6 & 99 & 92 & 4 \\ 4 & 5 & 6 & 58 & 62 & 8\end{array}$

$\begin{array}{rrrrr}5 & 5 & 6 & 77 & 70 \\ 6 & 5 & 6 & 0 & 5\end{array}$

$\begin{array}{rrrrrr}-6 & 5 & 6 & 56 & 44 & 9\end{array}$

$\begin{array}{llllll}-5 & 6 & 6 & 168 & 167 & 3 \\ -4 & 6 & 6 & 186 & 182 & 3\end{array}$

$\begin{array}{rrrrrr}-3 & 6 & 6 & 52 & 46 & 7 \\ -2 & 6 & 5 & 31 & 22 & 16\end{array}$

$\begin{array}{rrrrrr}-1 & 6 & 6 & 110 & 110 & 4\end{array}$

$\begin{array}{llllll}0 & 6 & 6 & 264 & 262\end{array}$

$\begin{array}{rrrrrr}1 & 6 & 6 & 111 & 113 & 4 \\ 2 & 6 & 6 & 0 & 21 & 1 \\ 3 & 6 & 6 & 0 & 1 & 1\end{array}$

$\begin{array}{rrrrrr}3 & 6 & 6 & 0 & 1 & 1 \\ 4 & 6 & 6 & 9 & 11 & 9\end{array}$

$\begin{array}{rrrrrr}4 & 6 & 6 & 9 & 11 & 9 \\ 5 & 6 & 6 & 70 & 69 & 8\end{array}$

$\begin{array}{rrrrrr}3 & 0 & 7 & 376 & 382 & 3 \\ 5 & 0 & 7 & 93 & 89 & 4\end{array}$

$\begin{array}{llllll}7 & 0 & 7 & 47 & 22 & 9\end{array}$

$\begin{array}{rrrrrr}-8 & 1 & 7 & 77 & 76 & 6 \\ -7 & 1 & 7 & 54 & 70 & 9\end{array}$

$\begin{array}{rrrrrr}-6 & 1 & 7 & 145 & 142 & 3\end{array}$

$\begin{array}{rrrrrr}-5 & 1 & 7 & 206 & 192 & 3 \\ -4 & 1 & 7 & 42 & 46 & 7\end{array}$

$\begin{array}{llllll}-3 & 1 & 7 & 48 & 47 & 5\end{array}$

$\begin{array}{rrrrrr}-2 & 1 & 7 & 391 & 40.2 & 3 \\ -1 & 1 & 7 & 9 & 38 & 9\end{array}$

$\begin{array}{rrrrrr}0 & 1 & 7 & 85 & 86 & 1 \\ 1 & 1 & 7 & 304 & 320 & 3\end{array}$

$2 \quad 1 \quad 7 \quad 66 \quad 76 \quad 4$

$\begin{array}{lllll}3 & 1 & 7 & 275 & 283\end{array}$

$\begin{array}{rrrrrr}4 & 1 & 7 & 114 & 107 & 3 \\ 5 & 1 & 7 & 49 & 45 & 9\end{array}$

$\begin{array}{llllll}6 & 1 & 7 & 88 & 81 & 5\end{array}$

$\begin{array}{rrrrrrr}7 & 1 & 7 & 62 & 59 & 8 \\ -8 & 2 & 7 & 71 & 64 & 7\end{array}$

$\begin{array}{rrrrr}-7 & 2 & 7 & 72 & 70 \\ -6 & 2 & 7 & 118 & 115\end{array}$

$\begin{array}{llllll}-5 & 2 & 7 & 147 & 140 & 3\end{array}$

$\begin{array}{llllll}-3 & 2 & 7 & 0 & 4 & 1\end{array}$

$\begin{array}{llllll}-2 & 2 & 7 & 102 & 108 & 2 \\ -1 & 2 & 7 & 250 & 240 & 3\end{array}$

$\begin{array}{llllll}0 & 2 & 7 & 391 & 413 & 7\end{array}$

$\begin{array}{rrrrrr}1 & 2 & 7 & 66 & 75 & 4 \\ 2 & 2 & 7 & 113 & 119 & 2\end{array}$

$\begin{array}{rrrrrr}3 & 2 & 7 & 51 & 55 & 7\end{array}$

$\begin{array}{lllll}4 & 2 & 7 & 83 & 87\end{array}$

$\begin{array}{rrrrrr}5 & 2 & 7 & 175 & 1.75 & 3 \\ 6 & 2 & 7 & 0 & 31 & 1\end{array}$

$\begin{array}{rrrrrr}7 & 2 & 7 & 32 & 3.9 & 20\end{array}$

$\begin{array}{rrrrrr}-8 & 3 & 7 & 119 & 126 & 5 \\ -7 & 3 & 7 & 67 & 64 & 7\end{array}$

$\begin{array}{rrrrrr}-6 & 3 & 7 & 167 & 157 & 3\end{array}$

$\begin{array}{rrrrrr}-5 & 3 & 7 & 35 & 21 & 13 \\ -4 & 3 & 7 & 161 & 159 & 2\end{array}$

$\begin{array}{llllll}-3 & 3 & 7 & 157 & 149 & 2\end{array}$

$\begin{array}{llllll}-2 & 3 & 7 & 409 & 424 & 3 \\ -1 & 3 & 7 & 115 & 123 & 2\end{array}$

$\begin{array}{rrrrrr}0 & 3 & 7 & 217 & 213 & 2\end{array}$

$\begin{array}{llllll}2 & 3 & 7 & 22 & 28 & 21\end{array}$

$\begin{array}{rrrrrr}3 & 3 & 7 & 0 & 40 & 1 \\ 4 & 3 & 7 & 49 & 26 & 8\end{array}$

$\begin{array}{rrrrrr}5 & 3 & 7 & 53 & 59 & 9 \\ 6 & 3 & 7 & 36 & 7 & 15\end{array}$

$\begin{array}{llllll}7 & 3 & 7 & 12 & 30 & 11\end{array}$

$\begin{array}{rrrrrr}-8 & 4 & 7 & 21 & 1 & 20\end{array}$

$\begin{array}{rrrrr}-7 & 4 & 7 & 82 & 83 \\ -6 & 4 & 7 & 101 & 101\end{array}$

$\begin{array}{lllll}-5 & 4 & 7 & 163 & 165\end{array}$

$\begin{array}{llllll}-4 & 4 & 7 & 129 & 133 & 3\end{array}$

$\begin{array}{lllllll}-3 & 4 & 7 & 135 & 139 & 3\end{array}$

$\begin{array}{llllll}-2 & 4 & 7 & 178 & 176 & 2\end{array}$

$\begin{array}{rrrrrr}-1 & 4 & 7 & 51 & 67 & 6 \\ 0 & 4 & 7 & 95 & 94 & 4\end{array}$

$\begin{array}{llllll}1 & 4 & 7 & 90 & 1.03 & 4\end{array}$

$\begin{array}{rrrrrr}2 & 4 & 7 & 101 & 99 & 3 \\ 3 & 4 & 7 & 91 & 94 & 4\end{array}$

$\begin{array}{rrrrrr}4 & 4 & 7 & 46 & 56 & 11\end{array}$

$\begin{array}{rrrrrr}5 & 4 & 7 & 103 & 93 & 4 \\ 6 & 4 & 7 & 0 & 5 & \end{array}$

$\begin{array}{rrrrrr}-7 & 5 & 7 & 0 & 13 & 1 \\ -6 & 5 & 7 & 16 & 51 & 1\end{array}$ 
Tabte 6 . Observed and calculated structure factors for 1

$\mathrm{h} k \mathrm{k}$ I IOFO IOFC $10 \mathrm{~s}$ h $\mathrm{k}$ l IOFO $10 \mathrm{FC} 10 \mathrm{~s}$ h $\mathrm{k}$ l 10Fo $10 \mathrm{FC} 10 \mathrm{~s}$

$\begin{array}{lllll}-5 & 5 & 7 & 111 & 110 \\ -4 & 5 & 7 & 0 & \end{array}$

$\begin{array}{rrrrrr}-4 & 5 & 7 & 0 & 9 & 1 \\ -3 & 5 & 7 & 14 & 32 & 14 \\ -2 & 5 & 7 & 15 & 51 & 14\end{array}$

$\begin{array}{rrrrrr}-2 & 5 & 7 & 15 & 51 & 14 \\ -1 & 5 & 7 & 133 & 134 & 3\end{array}$

$\begin{array}{rrrrr}0 & 5 & 7 & 58 & 61 \\ 1 & 5 & 7 & 180 & 183\end{array}$

$\begin{array}{rrrrrr}2 & 5 & 7 & 0 & 4 & 1 \\ 3 & 5 & 7 & 142 & 138 & \end{array}$

$\begin{array}{rrrrr}4 & 5 & 7 & 55 & 49 \\ 5 & 5 & 7 & 83 & 80\end{array}$

$\begin{array}{rrrrrr}5 & 5 & 7 & 25 & 6 & 24 \\ -6 & 6 & 7 & 87 & 89 & \end{array}$

$\begin{array}{rrrrr}-5 & 6 & 7 & 125 & 127\end{array}$

$\begin{array}{lllll}-4 & 6 & 7 & 178 & 173 \\ -3 & 6 & 7 & 113 & 116\end{array}$

$\begin{array}{rrrrrr}-2 & 6 & 7 & 27 & 21 & 27 \\ -1 & 6 & 7 & 54 & 39 & 7\end{array}$

$\begin{array}{llllll}0 & 6 & 7 & 199 & 205\end{array}$

\begin{tabular}{llllll}
1 & 6 & 7 & 162 & 169 \\
\hline & 6 & 7 & 0 & 0
\end{tabular}

$\begin{array}{rrrrr}2 & 6 & 7 & 0 & 12 \\ 3 & 6 & 7 & 106 & 106 \\ 1 & 6 & 7 & 9 & \end{array}$

$\begin{array}{rrrrrr}1 & 6 & 7 & 9 & 4 & 9 \\ 5 & 6 & 7 & 41 & 35 & 15\end{array}$

$\begin{array}{lllll}-5 & 7 & 7 & 64 & 55\end{array}$

$\begin{array}{rrrrr}-4 & 7 & 7 & 0 & 40 \\ -3 & 7 & 7 & 74 & 76 \\ -2 & 7 & 7 & 328 & 332\end{array}$

$\begin{array}{rrrrr}-1 & 7 & 7 & 0 & 17\end{array}$

$\begin{array}{rrrrr}0 & 7 & 7 & 9 & 15 \\ 1 & 7 & 7 & 0 & 24\end{array}$

$\begin{array}{rrrrr}2 & 7 & 7 & 180 & 184 \\ 3 & 7 & 7 & 59 & 50\end{array}$

$\begin{array}{rrrrrr}3 & 7 & 7 & 59 & 50 & 8 \\ 4 & 7 & 7 & 43 & 33 & 14\end{array}$

$\begin{array}{rrrrrr}-4 & 8 & 7 & 174 & 178 & 3 \\ -3 & 8 & 7 & 14 & 2 & 14\end{array}$

$\begin{array}{rrrrrr}-2 & 8 & 7 & 14 & 2 & 14 \\ -2 & 8 & 7 & 36 & 23 & 16 \\ -1 & 8 & 7 & 29 & 28 & 28\end{array}$

$\begin{array}{rrrrrr}0 & 8 & 7 & 222 & 235 & 2 \\ 1 & 8 & 7 & 105 & 93 & 5\end{array}$

$\begin{array}{rrrrrr}2 & 8 & 7 & 38 & 16 & 15 \\ -8 & 0 & 8 & 248 & 251 & 3\end{array}$

$\begin{array}{lllll}-8 & 0 & 8 & 248 & 251 \\ -5 & 0 & 8 & 266 & 274\end{array}$

$\begin{array}{lllll}-4 & 0 & 8 & 331 & 337\end{array}$

$\begin{array}{llllll}-2 & 0 & 8 & 40 & 6\end{array}$

$\begin{array}{lllll}2 & 0 & 8 & 169 & 161\end{array}$

$\begin{array}{rrrrrr}4 & 0 & 8 & 193 & 204 & \\ 6 & 0 & 8 & 33 & 13 & 15\end{array}$

$\begin{array}{lllll}-8 & 1 & 8 & 143 & 145\end{array}$

$\begin{array}{lllll}-5 & 1 & 8 & 316 & 320\end{array}$

$\begin{array}{rrrrr}-5 & 1 & 8 & 92 & 83 \\ -4 & 1 & 8 & 142 & 150\end{array}$

$\begin{array}{lllll}-3 & 1 & 8 & 536 & 503\end{array}$

$\begin{array}{lllll}-1 & 1 & 8 & 282 & 262\end{array}$

0 I $8 \quad 159 \quad 155$

$\begin{array}{lllll}3 & 1 & 8 & 265 & 270 \\ 2 & 1 & 8 & 156 & 161\end{array}$

$\begin{array}{lllll}3 & 1 & 8 & 70 & 63\end{array}$

$\begin{array}{rrrrr}4 & 1 & 8 & 89 & 88 \\ 5 & 1 & 8 & 200 & 204\end{array}$

$\begin{array}{lllll}6 & 1 & 8 & 127 & 127\end{array}$

$\begin{array}{rrrrr}7 & 1 & 8 & 0 & 69 \\ -8 & 2 & 8 & 154 & 154\end{array}$

$\begin{array}{rrrrr}-7 & 2 & 8 & 0 & 4\end{array}$

$\begin{array}{rrrrrr}-5 & 2 & 8 & 36 & 45 & 14 \\ -5 & 2 & 8 & 150 & 149 & 3\end{array}$

$\begin{array}{lllll}-4 & 2 & 8 & 176 & 141\end{array}$

$\begin{array}{rrrrr}-3 & 2 & 8 & 133 & 131 \\ -2 & 2 & 8 & 50 & 45\end{array}$

$\begin{array}{lllll}-1 & 2 & 8 & 455 & 457\end{array}$

$\begin{array}{rrrrr}0 & 2 & 8 & 80 & 76 \\ 1 & 2 & 8 & 51 & 57\end{array}$

$\begin{array}{rrrrr}2 & 2 & 8 & 107 & 99\end{array}$

$\begin{array}{lllll}3 & 2 & 8 & 357 & 354\end{array}$

$\begin{array}{rrrrrr}4 & 2 & 8 & 80 & 72 & 5 \\ 5 & 2 & 8 & 12 & 1 & 11\end{array}$

$\begin{array}{rrrrrr}5 & 2 & 8 & 91 & 85 & 5 \\ 7 & 2 & 8 & 46 & 43 & 12 \\ -8 & 3 & 8 & 9 & 65 & 9\end{array}$

$\begin{array}{rrrrr}-8 & 3 & 8 & 9 & 63 \\ -7 & 3 & 8 & 0 & 8\end{array}$

$\begin{array}{rrrrr}-6 & 3 & 8 & 0 & 15 \\ -5 & 3 & 8 & 165 & 165\end{array}$

$\begin{array}{lllll}-4 & 3 & 8 & 192 & 196 \\ -3 & 3 & 8 & 185 & 182\end{array}$

$\begin{array}{rrrrr}-2 & 3 & 8 & 77 & 81\end{array}$

$\begin{array}{rrrrr}-1 & 3 & 8 & 95 & 94 \\ 0 & 3 & 8 & 72 & 73\end{array}$ $\begin{array}{rrrrrr}1 & 3 & 8 & 419 & 436 & 3 \\ 2 & 3 & 8 & 18 & 28 & 18 \\ 3 & 3 & 8 & 59 & 65 & 6 \\ 4 & 3 & 8 & 80 & 77 & 5 \\ 5 & 3 & 8 & 93 & 99 & 5 \\ 6 & 3 & 8 & 42 & 39 & 12 \\ 7 & 3 & 8 & 0 & 3 & 1 \\ -7 & 4 & 8 & 9 & 25 & 9 \\ -6 & 4 & 8 & 329 & 319 & 4 \\ -5 & 4 & 8 & 156 & 152 & 3 \\ -4 & 4 & 8 & 183 & 170 & 3 \\ -3 & 4 & 8 & 113 & 110 & 3 \\ -2 & 4 & 8 & 306 & 300 & 4 \\ -1 & 4 & 8 & 208 & 212 & 3 \\ 0 & 4 & 8 & 21 & 14 & 20\end{array}$

$\begin{array}{rrrrrr}0 & 4 & 8 & 21 & 14 & 20 \\ 1 & 4 & 8 & 125 & 130 & 3\end{array}$

$\begin{array}{rrrrrr}2 & 4 & 8 & 84 & 81 & 4 \\ 3 & 4 & 8 & 153 & 150 & 3\end{array}$

$\begin{array}{rrrrrr}4 & 4 & 8 & 168 & 163 & 3 \\ 5 & 4 & 8 & 45 & 48 & 11\end{array}$

$\begin{array}{rrrrrr}6 & 4 & 8 & 0 & 2 & 1 \\ -7 & 5 & 8 & 29 & 51 & 29\end{array}$

$\begin{array}{rrrrrr}-7 & 5 & 8 & 29 & 51 & 29 \\ -6 & 5 & 8 & 121 & 113 & 4 \\ -5 & 5 & 8 & 64 & 59 & 7\end{array}$

$\begin{array}{rrrrrr}-4 & 5 & 8 & 387 & 383 & 4 \\ -3 & 5 & 8 & 55 & 63 & 8\end{array}$

$\begin{array}{rrrrrr}-3 & 5 & 8 & 55 & 63 & 8 \\ -2 & 5 & 8 & 148 & 140 & 3 \\ -1 & 5 & 8 & 79 & 75 & 4\end{array}$

$\begin{array}{rrrrrr}-1 & 5 & 8 & 79 & 75 & 4 \\ 0 & 5 & 8 & 125 & 115 & 2\end{array}$

$\begin{array}{rrrrrr}1 & 5 & 8 & 105 & 111 & 4 \\ 2 & 5 & 8 & 44 & 54 & \text { II }\end{array}$

$\begin{array}{rrrrrr}2 & 5 & 8 & 44 & 54 & 11 \\ 3 & 5 & 8 & 128 & 134 & 4\end{array}$

$\begin{array}{rrrrrr}4 & 5 & 8 & 0 & 36 & 1 \\ 5 & 5 & 8 & 0 & 25 & 1\end{array}$

$\begin{array}{rrrrrr}6 & 5 & 8 & 84 & 81 & 7 \\ -6 & 6 & 8 & 189 & 191 & 3\end{array}$

$\begin{array}{rrrrrr}-6 & 6 & 8 & 189 & 191 & 3 \\ -5 & 6 & 8 & 141 & 147 & 4 \\ -4 & 6 & 8 & 69 & 74 & 7\end{array}$

$\begin{array}{rrrrrr}-4 & 6 & 8 & 69 & 74 & 7 \\ -3 & 6 & 8 & 28 & 40 & 28\end{array}$

$\begin{array}{rrrrrr}-2 & 6 & 8 & 95 & 98 & 4 \\ -1 & 6 & 8 & 261 & 269 & 3\end{array}$

$\begin{array}{rrrrrr}0 & 6 & 8 & 105 & 111 & 3 \\ 1 & 5 & 8 & 17 & 11 & 16\end{array}$

$\begin{array}{rrrrrr}1 & 5 & 8 & 17 & 11 & 16 \\ 2 & 6 & 8 & 0 & 25 & 1 \\ 3 & 6 & 8 & 162 & 160 & 3\end{array}$

$\begin{array}{rrrrrr}3 & 6 & 8 & 162 & 160 & 3 \\ 4 & 6 & 8 & 23 & 12 & 23\end{array}$

$\begin{array}{rrrrrr}5 & 6 & 8 & 46 & 41 & 12 \\ -5 & 7 & 8 & 59 & 62 & 9\end{array}$

$\begin{array}{rrrrrr}-5 & 7 & 8 & 59 & 62 & 9 \\ -4 & 7 & 8 & 57 & 46 & 8\end{array}$

$\begin{array}{rrrrrr}-3 & 7 & 8 & 322 & 330 & 4 \\ -2 & 7 & 8 & 52 & 71 & 10\end{array}$

$\begin{array}{llllll}-1 & 7 & 8 & 36 & 39 & 15\end{array}$

$\begin{array}{rrrrrr}0 & 7 & 8 & 51 & 57 & 8 \\ 1 & 7 & 8 & 196 & 193 & 3\end{array}$

$\begin{array}{rrrrrr}2 & 7 & 8 & 65 & 64 & 8 \\ 3 & 7 & 8 & 56 & 54 & 9\end{array}$

$\begin{array}{rrrrrr}4 & 7 & 8 & 22 & 18 & 21\end{array}$

$\begin{array}{rrrrrr}-3 & 8 & 8 & 0 & 8 & 1 \\ -2 & 8 & 8 & 51 & 31 & 10 \\ -1 & 8 & 8 & 186 & 186 & 3\end{array}$

$\begin{array}{rrrrrr}0 & 8 & 8 & 0 & 8 & 1 \\ 1 & 8 & 8 & 56 & 61 & 10\end{array}$

$\begin{array}{rrrrrr}1 & 8 & 8 & 56 & 61 & 10\end{array}$

$\begin{array}{rrrrrr}-7 & 0 & 9 & 0 & 5 & 1 \\ -5 & 0 & 9 & 230 & 234 & 3\end{array}$

$\begin{array}{rrrrrr}-3 & 0 & 9 & 24 & 16 & 18 \\ -1 & 0 & 9 & 1244 & 1228 & 3\end{array}$

$\begin{array}{llllll}1 & 0 & 9 & 224 & 222 & 3 \\ 3 & 0 & 9 & 243 & 247 & 3\end{array}$

$\begin{array}{rrrrrr}3 & 0 & 9 & 243 & 247 & 3 \\ 5 & 0 & 9 & 28 & 2 & 27 \\ 7 & 0 & 9 & 52 & 73 & 10\end{array}$

$\begin{array}{rrrrrr}7 & 0 & 9 & 52 & 73 & 10 \\ -8 & 1 & 9 & 149 & 156 & 4\end{array}$

$\begin{array}{rrrrrr}-7 & 1 & 9 & 0 & 28 & 1 \\ -6 & 1 & 9 & 77 & 87 & 6\end{array}$

$\begin{array}{rrrrr}-6 & 1 & 9 & 77 & 87 \\ -5 & 1 & 9 & 154 & 157\end{array}$

$\begin{array}{lllll}-4 & 1 & 9 & 152 & 157\end{array}$

$\begin{array}{rrrrr}-3 & 1 & 9 & 502 & 503 \\ -2 & 1 & 9 & 78 & 79\end{array}$

$\begin{array}{rrrrrr}-1 & 1 & 9 & 78 & 79 & 3 \\ -1 & 1 & 9 & 75 & 77 & 3\end{array}$

$\begin{array}{llllll}0 & 1 & 9 & 168 & 168 & 1\end{array}$

$\begin{array}{llllll}1 & 1 & 9 & 505 & 517 & 3 \\ 2 & 1 & 9 & 146 & 149 & 2\end{array}$

$\begin{array}{rrrrrr}3 & 1 & 9 & 22 & 3 & 21 \\ 4 & 1 & 9 & 99 & 104 & 4\end{array}$

$\begin{array}{rrrrrr}4 & 1 & 9 & 99 & 104 & 4 \\ 5 & 1 & 9 & 61 & 57 & 7 \\ 6 & 1 & 9 & 29 & 38 & 29\end{array}$

\begin{tabular}{rrrrrr}
7 & 1 & 9 & 29 & 38 & 29 \\
\hline & 1 & 9 & 56 & 39 & 9
\end{tabular}

$\begin{array}{llllll}-8 & 2 & 9 & 117 & 121 & 4\end{array}$

$\begin{array}{llllll}-7 & 2 & 9 & 176 & 172 & 3 \\ -6 & 2 & 9 & 225 & 225 & 3\end{array}$

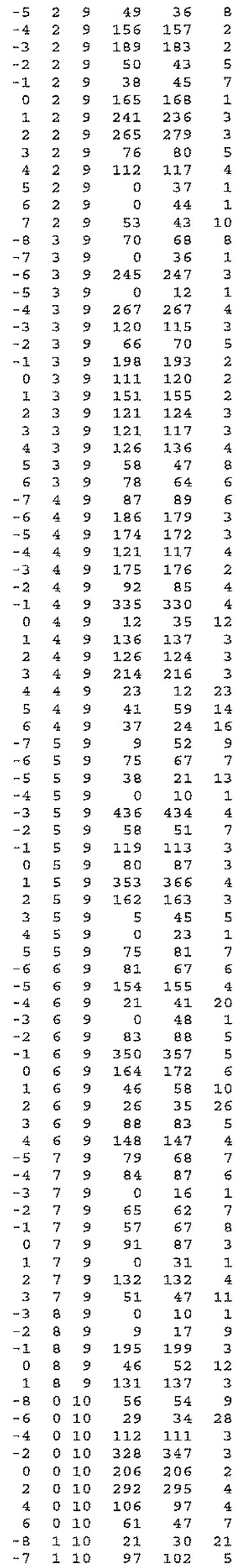

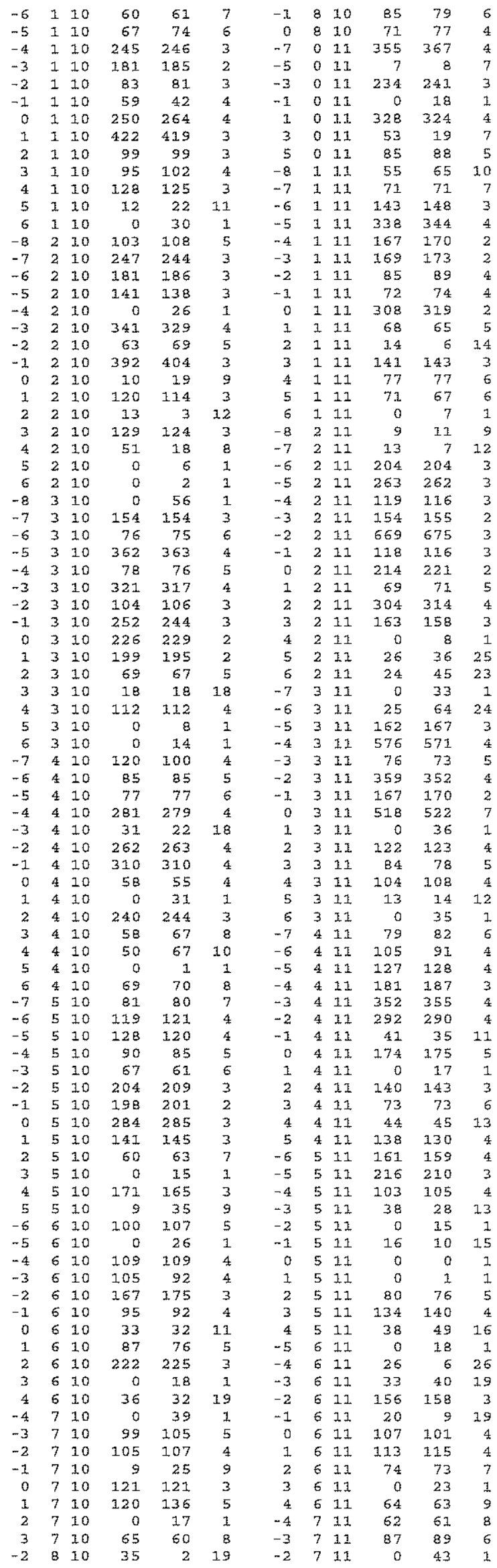


Table 6. Observed and calculated structure factors for 1

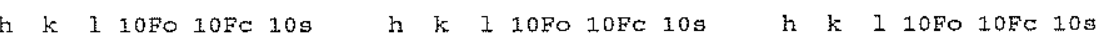
h $k$ I LOFO 10FC 10s

h $k 1$ LOFO 1OFC $10 \mathrm{~s}$

\begin{tabular}{|c|c|c|c|c|c|c|c|c|}
\hline-1 & 7 & & 57 & 43 & 8 & -1 & 7 & 12 \\
\hline 0 & 7 & 11 & 200 & 205 & 4 & 0 & 7 & 12 \\
\hline 1 & 7 & 11 & 0 & 19 & 1 & 3 & 7 & 12 \\
\hline 2 & 7 & 11 & 58 & 50 & 9 & -7 & 0 & 13 \\
\hline-8 & 0 & 12 & 64 & 62 & 8 & -5 & 0 & 13 \\
\hline-6 & 0 & 12 & 144 & 148 & 3 & -3 & 0 & 13 \\
\hline$-\frac{1}{2}$ & 0 & 12 & 430 & 441 & 4 & -1 & 0 & 13 \\
\hline-2 & 0 & 12 & 85 & 97 & 4 & 1 & $a$ & 13 \\
\hline 0 & 0 & 12 & 295 & 296 & 3 & 3 & 0 & 13 \\
\hline 2 & 0 & 12 & 161 & 158 & 2 & 5 & 0 & 13 \\
\hline 4 & 0 & 12 & 0 & 45 & 1 & -7 & 1 & 13 \\
\hline 6 & 0 & 12 & 39 & 4 & 15 & -6 & I. & 13 \\
\hline-8 & 1 & 12 & 0 & 8 & 1 & -5 & 1 & 13 \\
\hline-7 & 1 & 12 & 0 & 7 & 1 & -4 & $I$ & 13 \\
\hline-5 & I & I2 & 106 & 101 & 4 & -3 & 1 & 13 \\
\hline-5 & 1 & 12 & 242 & 240 & 3 & -2 & 1 & 13 \\
\hline-4 & $I$ & 12 & 76 & 70 & 5 & -1 & 1 & 13 \\
\hline-3 & 1 & 12 & 128 & 120 & 3 & 0 & 1 & 13 \\
\hline-2 & 1 & 12 & 260 & 274 & 4 & 1 & 1 & 13 \\
\hline-1 & 1 & 12 & 136 & 130 & 3 & 2 & 1 & 13 \\
\hline 0 & 1 & 12 & 236 & 244 & 2 & 3 & 1 & 13 \\
\hline 1 & 3 & 12 & 100 & 101 & 4 & 4 & 1 & 13 \\
\hline 2 & 1 & 12 & 175 & 179 & 2 & 5 & 1 & 13 \\
\hline 3 & 1 & 12 & 309 & 304 & 4 & -7 & 2 & 13 \\
\hline 4 & 1 & 12 & 65 & 65 & 7 & -6 & 2 & 13 \\
\hline 5 & 1. & 12 & 0 & 25 & 1 & -5 & 2 & 13 \\
\hline 6 & 1 & 12 & 20 & 1 & 19 & -4 & 2 & 13 \\
\hline-8 & 2 & 12 & 22 & 22 & 22 & -3 & 2 & 13 \\
\hline-7 & 2 & 12 & 89 & 93 & 5 & -2 & 2 & 13 \\
\hline-6 & 2 & 12 & 147 & 144 & 3 & -1 & 2 & 13 \\
\hline-5 & 2 & 12 & 112 & 110 & 4 & 0 & 2 & 13 \\
\hline-4 & 2 & 12 & 66 & $6 \frac{A}{x}$ & 6 & 1 & 2 & 13 \\
\hline-3 & 2 & 12 & 150 & 154 & 3 & 2 & 2 & 13 \\
\hline-2 & 2 & 12 & 107 & 111 & 4 & 3 & 2 & 13 \\
\hline-1 & 2 & 12 & 0 & 25 & I & 4 & 2 & 13 \\
\hline 0 & 2 & 12 & 216 & 226 & 5 & 5 & 2 & 13 \\
\hline I & 2 & 12 & 220 & 234 & 3 & -7 & 3 & 13 \\
\hline 2 & 2 & 12 & 110 & 112 & 4 & -6 & 3 & 13 \\
\hline 3 & 2 & 12 & 118 & 115 & 4 & -5 & 3 & 13 \\
\hline 4 & 2 & 12 & 53 & 48 & 8 & -4 & 3 & 13 \\
\hline 5 & 2 & 12 & 218 & 214 & 3 & -3 & 3 & 13 \\
\hline 5 & 2 & 12 & 29 & 24 & 29 & -2 & 3 & 13 \\
\hline-7 & 3 & 12 & 0 & 5 & 1 & -1 & 3 & 13 \\
\hline-5 & 3 & 12 & 0 & 49 & 1 & 0 & 3 & 13 \\
\hline-5 & 3 & 12 & 0 & 15 & 1 & 1 & 3 & 13 \\
\hline-4 & 3 & 12 & 232 & 230 & 3 & 2 & 3 & 13 \\
\hline-3 & 3 & 12 & 266 & 266 & 3 & 3 & 3 & 13 \\
\hline-2 & 3 & 12 & 95 & 92 & 4 & 4 & 3 & 13 \\
\hline-3 & 3 & 12 & 0 & 2 & 1 & 5 & 3 & 13 \\
\hline 0 & 3 & 12 & 207 & 209 & 2 & -6 & 4 & 13 \\
\hline 1 & 3 & 12 & 73 & 72 & 6 & -5 & 4 & 13 \\
\hline 2 & 3 & 12 & 37 & 21 & 13 & -4 & 4 & 13 \\
\hline 3 & 3 & 12 & 207 & 205 & 3 & -3 & 4 & 13 \\
\hline 4 & 3 & 12 & 24 & 28 & 23 & -2 & 4 & 13 \\
\hline 5 & 3 & 12 & 98 & 104 & 5 & -1 & 4 & 13 \\
\hline-7 & 4 & 12 & 57 & 26 & 9 & 0 & 4 & 13 \\
\hline-6 & 4 & 12 & 86 & 81 & 6 & 1 & 4 & 13 \\
\hline-5 & 4 & 12 & 72 & 73 & 6 & 2 & 4 & 13 \\
\hline-4 & 4 & 12 & 30 & 19 & 19 & 3 & 4 & 13 \\
\hline-3 & 4 & 12 & 26 & 9 & 25 & 4 & 4 & 13 \\
\hline-2 & 4 & 12 & 547 & 542 & 4 & -6 & 5 & 13 \\
\hline-1 & 4 & 12 & $a$ & 30 & 1 & -5 & 5 & 13 \\
\hline 0 & 4 & 12 & 92 & 93 & 3 & -4 & 5 & 13 \\
\hline 1 & 4 & 12 & 184 & 183 & 3 & -3 & 5 & 13 \\
\hline 2 & 4 & 12 & 152 & 162 & 3 & -2 & 5 & 13 \\
\hline 3 & 1 & 12 & 71 & 83 & 7 & -1 & 5 & 13 \\
\hline 4 & 4 & 12 & 30 & 61 & 29 & 0 & 5 & 13 \\
\hline 5 & 4 & 12 & 93 & 93 & $\sigma$ & 1 & 5 & 13 \\
\hline-6 & 5 & 12 & 183 & 183 & 3 & 2 & 5 & 13 \\
\hline-5 & 5 & 12 & 0 & 32 & 1 & 3 & 5 & 13 \\
\hline-4 & 5 & 12 & 279 & 274 & 4 & -5 & 6 & 13 \\
\hline-3 & 5 & 12 & 41 & 21 & 11 & -4 & 6 & 13 \\
\hline-2 & 5 & 12 & 168 & 164 & 3 & -3 & 6 & 13 \\
\hline-1 & 5 & 12 & 69 & 69 & 6 & -2 & 6 & 1.3 \\
\hline 0 & 5 & 12 & 313 & 315 & 9 & -1 & 6 & 13 \\
\hline 3 & 5 & 12 & 62 & 54 & 7 & 0 & 6 & 13 \\
\hline 2 & 5 & 12 & 70 & 68 & 7 & 1 & 6 & 13 \\
\hline 3 & 5 & 12 & 47 & 40 & 11 & 2 & 6 & 1.3 \\
\hline 4 & 5 & 12 & 22 & 12 & 21 & -3 & 7 & 1.3 \\
\hline-5 & 6 & 12 & 64 & 67 & 8 & -2 & 7 & 13 \\
\hline-4 & 6 & 12 & 239 & 243 & 3 & -1 & 7 & 13 \\
\hline-3 & 6 & 12 & 0 & 32 & 1 & 0 & 7 & 13 \\
\hline-2 & 6 & 12 & 132 & 135 & 4 & -6 & 0 & 14 \\
\hline-1 & 6 & 12 & 90 & 83 & 5 & -4 & 0 & 14 \\
\hline 0 & 6 & 12 & 37 & 12 & 14 & -2 & 0 & 14 \\
\hline 1 & 6 & 12 & 143 & 139 & 4 & 0 & 0 & 14 \\
\hline 2 & 6 & 12 & $10 \frac{4}{2}$ & 110 & & 2 & 0 & 14 \\
\hline 3 & 6 & 12 & 28 & 3 & 27 & 4 & 0 & 14 \\
\hline-3 & 7 & 12 & 53 & 61 & 10 & -7 & I & 14 \\
\hline & 7 & 12 & 0 & 16 & 1 & -6 & I & 14 \\
\hline
\end{tabular}


Table 6. Observed and calculated structure factors for 1

Page 5
h $k$ l 10FO 10Ec 10s
$\mathrm{h} k \mathrm{k}$ 10Fo $10 \mathrm{Fc}$ tos
$\mathrm{h} \mathrm{k} 1$ 10Fo $10 \mathrm{FC} 10 \mathrm{~s}$
h $\mathrm{k} 1$ 10FO IOFC $10 \mathrm{~s}$
$\begin{array}{rrrrrr}-2 & 3 & 19 & 0 & 17 & 1 \\ -1 & 3 & 19 & 94 & 95 & 5\end{array}$
$\begin{array}{rrrrrr}-2 & 0 & 20 & 9 & 30 & 9 \\ 0 & 0 & 20 & 5 & 11 & 5 \\ -3 & 1 & 20 & 102 & 98 & 5\end{array}$
$\begin{array}{rrrrrr}-2 & 1 & 20 & 0 & 7 & 1 \\ -1 & 1 & 20 & 165 & 157 & 4 \\ 0 & 1 & 20 & 43 & 37 & 21\end{array}$
$\begin{array}{rrrrrr}-2 & 2 & 20 & 68 & 69 & B \\ -1 & 2 & 20 & 129 & 120 & 4\end{array}$

h $\mathrm{k} l$ IOFO IDFC $10 \mathrm{~s}$ 